\title{
DIFFUSION MEDIATED TRANSPORT WITH A LOOK AT MOTOR PROTEINS
}

\author{
STUART HASTINGS *, DAVID KINDERLEHRER †, AND J. BRYCE MCLEOD ‡
}

\begin{abstract}
In this note we discuss transport properties of weakly coupled parabolic systems of evolution equations. These arise in the study of molecular motors, like conventional kinesin, which are responsible for eukaryotic intracellular transport. It falls under the rubric of what we call diffusion mediated transport. Diffusion mediated transport generally concerns directed transport or oriented fluctuations of a system with a high degree of randomness and requires special collaboration among its various elements to achieve. We discuss how this plays out in multiple state systems.
\end{abstract}

1. Introduction. Intracellular transport in eukarya is attributed to motor proteins that transduce chemical energy into conformational changes which lead to directed mechanical motion. Nanoscale motors like kinesins tow organelles and other cargo on microtubules or filaments, have a role separating the mitotic spindle during the cell cycle, and perform many other functions. The simplest description gives rise to a weakly coupled system of evolution equations. The transport process, to the mind's eye, is comparable to a biased coin toss. This intuition may be confirmed by a careful analysis of the cooperative effects among the conformational changes and the potentials. Two models illustrating this are a two state system and a larger system intended to account for the neck linker apparatus. We then discuss how collaboration may fail when connectivity among the elements comprising the network is disrupted, as in the case of spacers added to the neck-linker. Most of these remarks are about [11].

Suppose that $\rho_{1}, \ldots, \rho_{n}$ are partial probability densities defined on the unit interval $\Omega=(0,1)$ satisfying

$$
\begin{aligned}
\frac{d}{d x}\left(\sigma \frac{d \rho_{i}}{d x}+\psi_{i}^{\prime} \rho_{i}\right)+ & \sum_{j=1, \ldots, n} a_{i j} \rho_{j}=0 \text { in } \Omega \\
\sigma \frac{d \rho_{i}}{d x}+\psi_{i}^{\prime} \rho_{i} & =0 \text { on } \partial \Omega, i=1, \ldots n, \\
\rho_{i} \geqq 0 \text { in } \Omega, \int_{\Omega}\left(\rho_{1}+\cdots+\rho_{n}\right) d x & =1 .
\end{aligned}
$$

* Department of Mathematics, University of Pittsburgh, Pittsburgh, PA 15260, USA, email: sph+@pitt.edu

${ }^{\dagger}$ Center for Nonlinear Analysis and Department of Mathematical Sciences, Carnegie Mellon University, Pittsburgh, PA 15213, email: davidk@cmu.edu Research supported by NSF DMS 0305794 and DMS 0405343

${ }^{\ddagger}$ Department of Mathematics, University of Pittsburgh, Pittsburgh, PA 15260 email: mcleod@pitt.edu 
Here $\sigma>0, \psi_{1}, \ldots, \psi_{n}$ are smooth non-negative functions of period $1 / N$, and $A=\left(a_{i j}\right)$ is a smooth rate matrix of period $1 / N$, that is, $a_{i j}$ are $1 / N$-periodic functions with

$$
\begin{gathered}
a_{i i} \leqq 0, a_{i j} \geqq 0 \text { for } i \neq j \text { and } \\
\sum_{i=1, . ., n} a_{i j}=0, j=1, \ldots, n
\end{gathered}
$$

Note that for $\tau>0$ small, the matrix $P=\mathbf{1}+\tau A$ is a probability matrix. The system (1.1) is the stationary equations of the evolution system

$$
\begin{aligned}
\frac{\partial \rho_{i}}{\partial t}=\frac{\partial}{\partial x}\left(\sigma \frac{\partial \rho_{i}}{\partial x}+\psi_{i}^{\prime} \rho_{i}\right)+ & \sum_{j=1, \ldots, n} a_{i j} \rho_{j}=0 \text { in } \Omega, t>0 \\
\sigma \frac{\partial \rho_{i}}{\partial x}+\psi_{i}^{\prime} \rho_{i} & =0 \text { on } \partial \Omega, t>0, i=1, \ldots n, \\
\rho_{i} \geqq 0 \text { in } \Omega, \int_{\Omega}\left(\rho_{1}+\cdots+\rho_{n}\right) d x & =1, t>0 .
\end{aligned}
$$

Before discussing the result further, let us discuss what we intend by transport. In a chemical or conformational change process, a reaction coordinate (or coordinates) must be specified. This is the independent variable. In a mechanical system, it is usually evident what this coordinate must be. In our situation, even though both conformational change and mechanical effects are present, it is natural to specify the distance along the motor track, the microtubule, here the interval $\Omega$, as the independent variable. We interpret the migration of density during the evolution to one end of the track as evidence of transport.

Transport results from functional relationships in this system. A straightforward way to approach this issue is inspection of the dissipation principle which gives an implicit scheme for its solution. This implicit scheme is based on a Monge-KantorovichWasserstein metric, which, we recall, may be defined by

$$
d\left(f, f^{*}\right)^{2}=\inf _{\mathbf{P}} \int_{\Omega \times \Omega}|x-y|^{2} d p(x, y)
$$

where $\mathbf{P}$ denotes the set of joint distributions for $f$ and $f^{*}$, nonnegative densities with the same total mass. Let 


$$
\begin{aligned}
& F(\eta)=\int_{\Omega} \sum_{i=1, \ldots, n}\left\{\psi_{i} \eta_{i}+\sigma \eta_{i} \log \eta_{i}\right\} d x \\
& \eta_{i} \geqq 0 \text { and } \int_{\Omega} \sum_{i=1, \ldots, n} \eta_{i} d x=1
\end{aligned}
$$

denote the free energy. Now given a state $\rho^{*}$, determine its successor state $\rho$ by resolving the variational principle

$$
\begin{array}{r}
\frac{1}{2 \tau} \sum_{i=1, \ldots, n} d\left(\rho_{i},\left(P \rho^{*}\right)_{i}\right)^{2}+F(\rho)=\min , \\
\int_{\Omega} \rho_{i} d x=\int_{\Omega}\left(P \rho^{*}\right)_{i} d x
\end{array}
$$

where $P$ is the probability matrix above. Below we address why we call this a dissipation principle. For the moment, determine an implicit scheme by the rule: given $\rho^{(k-1)}$, set $\rho^{*}=\rho^{(k-1)}$ and $\rho^{k}=\rho$ in (1.6), then set

$$
\rho^{\tau}=\rho^{k}, k \tau \leqq t<(k+1) \tau .
$$

Finally, let $\tau \rightarrow 0$. Then $\rho^{\tau} \rightarrow \rho$, the solution of $(1.3)$ in $\Omega \times(0, T)$ for any $T<\infty$.

The interpretation of (1.6) as a dissipation principle is given in [5] and is based in part on the Benamou and Brenier [3] result that in (1.4)

$$
\frac{1}{\tau} d\left(f, f^{*}\right)^{2}=\min \int_{0}^{\tau} \int_{\Omega} v^{2} f d x d t
$$

where the minimum is taken over families $f(x, t), 0 \leqq t \leqq \tau$, of deformations satisfying

$$
\begin{aligned}
& \frac{\partial f}{\partial t}+\frac{\partial}{\partial x}(v f)=0 \text { in } \Omega \times(0, \tau), \\
& f(x, 0)=f^{*}(x) \text { and } f(x, \tau)=f(x) .
\end{aligned}
$$

The right hand side of (1.7) is the minimum dissipation of an ensemble of highly damped particles initially distributed by $f^{*}$ and terminally distributed by $f$, expressed in an eulerian frame. So the minimum energy budget in moving the system from $\rho^{*}$ to $\rho$ is given by the variational problem (1.6). It has the merit of isolating the free energy, the dissipation, and the conformational change. This is modulo some modelling of the entropic contribution, for which we have adopted combinatorial indeterminacy, the simplest possible choice. We may also interpret this as representing a collection of molecular motors as a conformation changing ensemble of spring-mass-dashpots. Here we are glossing over the many issues present in modelling small scale systems, where mechanics and chemistry or conformational changes themselves operate at disparate time and length scales. 
Our first statement is that the stationary solution of the system (1.1), which we denote by $\rho^{\sharp}$, is globally stable: given any solution $\rho(x, t)$ of $(1.3)$,

$$
\rho(x, t) \rightarrow \rho^{\sharp}(x) \text { as } t \rightarrow \infty
$$

So the migration of density, constituting transport, we referred to previously may be ascertained by inspection of $\rho^{\sharp}$. In the sequel, we simply set $\rho=\rho^{\sharp}$.

We shall characterize transport properties of (1.1) by decay properties of $\rho$. If the preponderance of mass of $\rho$ is distributed at one end of the track, then transport is present. Our main result, stated precisely later, is that with suitable collaboration among the potentials $\psi_{1}, \ldots, \psi_{n}$ and the rate matrix $A$, there are constants $K$ and $M$, independent of $\sigma$, such that

$$
\sum_{i=1}^{n} \rho_{i}\left(x+\frac{1}{N}\right) \leqq K e^{-\frac{M}{\sigma}} \sum_{i=1}^{n} \rho_{i}(x), x \in \Omega, x<1-\frac{1}{N}
$$

for sufficiently small $\sigma>0$.

Our objective in this note is to explain what we have found to be the suitable collaboration mentioned above. A first possibility is to ask simply for the equilibrium configuration, associated to (1.5), that is, its minimum energy configuration, given by

$$
\begin{gathered}
\rho_{i}^{\sharp}(x)=\frac{1}{Z} e^{-\frac{\psi_{i}(x)}{\sigma}}, x \in \Omega, i=1, \ldots, n \\
Z=\int_{\Omega}\left(e^{-\frac{\psi_{1}(x)}{\sigma}}+\cdots+e^{-\frac{\psi_{n}(x)}{\sigma}}\right) d x,
\end{gathered}
$$

which is $1 / N$-periodic in $\Omega$. This solution does not exhibit transport and for it to be a solution of (1.1) means that the equations decouple and

$$
A \rho=0 \text { in } \Omega .
$$

This is a detailed balance situation, separately for the Markov Process described by the $n$ individual Fokker-Planck Equations and for the Markov chain with transition matrix $P$. So, to favor transport, equilibrium should not be attained and detailed balance broken. But failure of detailed balance is far from sufficient to produce transport. We illustrate this in Figure 1.1 where solutions of a two species system for two arrangements of symmetric potential wells are shown. The matrix $A$ was chosen constant, which favors a maximum amount of transport. Detailed balance fails for the solutions. Asymmetry of the potentials was suggested early in the study of motor proteins, cf. [2], [17], and it is known that the microtubules and actin filiments which host motors are polarized, is suspected to play a role here. Asymmetry itself is insufficient. In Figure 1.2, asymmetric potentials differ from each other by a slight shift, actually $1 / 8$ period, and there is no noticable transport in the solution.

If we adopt the pragmatic notion that in a two species system, the two species function in the same way, we are led to interdigitated potentials $\psi_{j}$ of the form in Figure 1.3. This is not a reason, of course. We discuss this further below.

We are led to the intriguing question of the relationship between the $\psi_{j}$ and $A$. Even under the most most propitious circumstances, one may always add to the system 

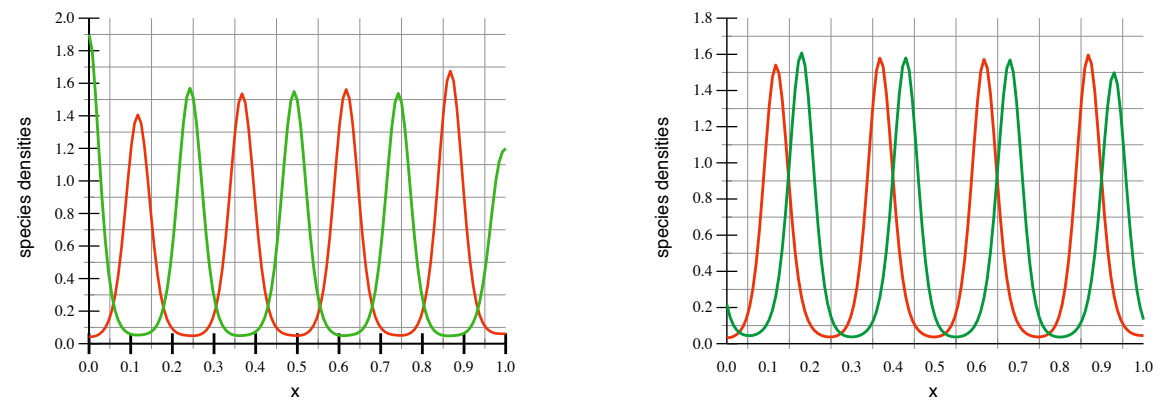

FIG. 1.1. Solutions of (1.1) for two state systems with symmetric potentials placed symmetrically (left) and symmetric potentials placed asymmetrically (right) showing lack of transport of density. A was chosen constant to optimize the possibility of transport. Detailed balance is not satisfied by the solutions

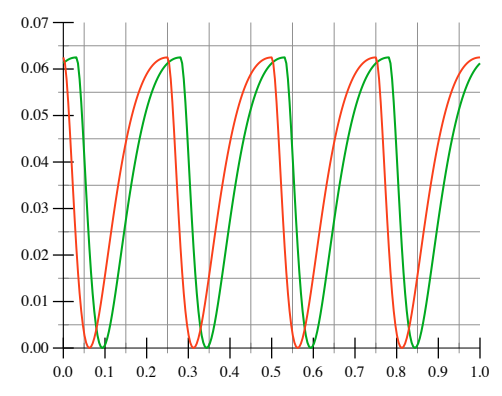

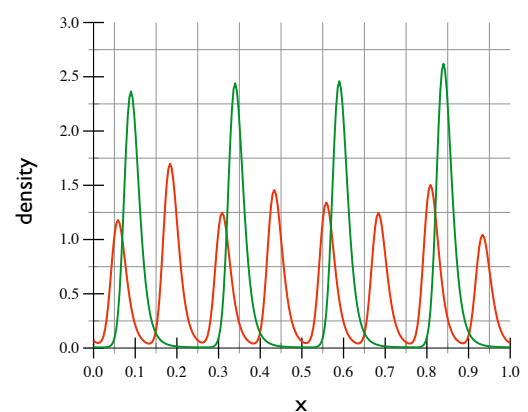

FIG. 1.2. Slightly shifted asymmetric wells, left, and the solutions of (1.1), right, illustrating lack of transport. The matrix A was chosen to optimize transport possibilities.

independent uncoupled equations. So it is necessary, in view of (1.2), that

$$
a_{i i} \not \equiv 0 \text { in } \Omega
$$

But where and how? What are the possibilities here? The basic mechanism of diffusional transport is that mass is transported to specific sites determined by minima and local minima of the potential. For directed transport, to the left toward $x=0$, for example, in any subinterval of a period interval, there should be some $\psi_{i}$ which is increasing. This explains the result shown in Figure 1.2, where the potentials are asymmetric and transport is not present. Moreover, some interchange must take place: mass in states associated to each of the $\psi_{j}$ which is decreasing should have the opportunity to change to the $i^{t h}$-state. This is reminiscent of an ergodic hypothesis. It does not say that all states are connected, but it will be a very strong condition since it will be required to hold near all the minima of all of the potentials. In the the neck linker example we have mentioned, the condition fails and so does the conclusion 


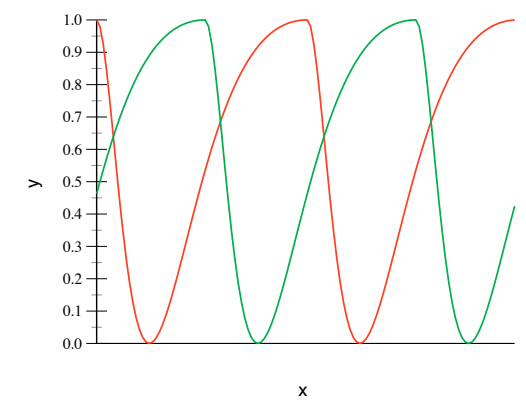

FIG. 1.3. Asymmetric periodic potentials symmetrically interdigitated, the configuration which promotes transport

of our theorem. We give a more precise description below.

We have, in the above discussion, tacitly assumed the existence of a positive solution of (1.1). In fact, under suitable conditions on the $a_{i j}$, we can assert not only the existence of a solution of (1.1), without assuming a priori its positivity, but also that it is unique and necessarily positive. Sufficient conditions for this are (1.2) together with the hypothesis that, with $P_{\tau}=\mathbf{1}+\tau A$ the probability matrix already mentioned, that

$$
\begin{aligned}
& a_{i j} \not \equiv 0 \text { in } \Omega, \text { or more generally, } \\
& Q_{\tau}=\int_{\Omega} P_{\tau}(x) d x \text { is ergodic, }
\end{aligned}
$$

i.e., $Q_{\tau}^{k}$ has all positive entries for some integer $k$.

Further, we can prove that the positive stationary solution, $\rho^{\dagger}(x)$ say, of (1.1) is globally stable in the sense that given any initial data $f(x)$ with corresponding solution $\rho(x, t)$ of $(1.3)$ there exist $c>0$ and $\omega>0$ such that

$$
\rho(x, t)=c \rho^{\dagger}(x)+O\left(e^{-\omega t}\right) \text { as } t \rightarrow \infty
$$

There are various ways of proving this, they all depend on ideas from positive operators. In these frameworks, we define the operator $e^{S}$ by expressing the solution $\rho$ of (1.3) by

$$
\rho(x, t)=e^{t S} f(x), x \in \Omega, t>0 .
$$

From the maximum principle, eg. [25], this is a positive operator. This enables us to apply a variant of the Krein-Rutman Theorem, the appropriate generalization of the Perron-Frobenius Theorem for matrices, to understand the spectrum of $S$. We refer to [4], [11] for additional details. In [23], Perthame succeeds in constructing an appropriate entropy function for the system, at least when the number of species $n=2$. 
We take this opportunity to thank our collaborators Michel Chipot and Michal Kowalczyk for their help. We also thank Bard Ermentrout, Michael Grabe, and Jonathan Rubin, and the Mathematical Biology Seminar of the University of Pittsburgh for generosity of spirit and their interest.

2. Main transport result. Here we state our main result about transport in multiple state systems.

THEOREM 2.1. Suppose that $\rho$ is a positive solution of (1.1), where the coefficients $a_{i j}, i, j=1, \ldots, n$ and the $\psi_{i}, i=1, \ldots, n$ are smooth and $1 / N$-periodic in $\bar{\Omega}$. Suppose that (1.2) holds and also that the following conditions are satisfied.

(i) Each $\psi_{i}^{\prime}$ has only a finite number of zeros in $\bar{\Omega}$.

(ii) There is some interval in which $\psi_{i}^{\prime}>0$ for all $i=1, \ldots, n$.

(iii) In any interval in which no $\psi_{i}^{\prime}$ vanishes, $\psi_{j}^{\prime}>0$ in this interval for at least one $j$.

(iv) If $I,|I|<1 / N$, is an interval in which $\psi_{i}^{\prime}>0$ for $i=1, . ., p$ and $\psi_{i}^{\prime}<0$ for $i=p+1, . ., n$, and $a$ is a zero of at least one of the $\psi_{k}^{\prime}$ which lies within $\epsilon$ of the right-hand end of $I$, then for $\epsilon$ sufficiently small, there is at least one index $i, i=1, \ldots, p$, with $a_{i j}>0$ in $(a-\eta, a)$ for some $\eta>0$, all $j=p+1, . ., n$.

Then, there exist positive constants $K, M$ independent of $\sigma$ such that

$$
\sum_{i=1}^{n} \rho_{i}\left(x+\frac{1}{N}\right) \leqq K e^{-\frac{M}{\sigma}} \sum_{i=1}^{n} \rho_{i}(x), x \in \Omega, x<1-\frac{1}{N}
$$

for sufficiently small $\sigma$.

We give a sketch of the proof intended to highlight the role of $(i v)$. Let $\lambda=1 / N$ denote the period. Adding the equations in (1.1) gives

$$
\frac{d}{d x} \sum_{i=1, \ldots, n}\left(\sigma \frac{d \rho_{i}}{d x}+\psi_{i}^{\prime} \rho_{i}\right)=0 \text { in } \Omega
$$

and by the boundary condition

$$
\sum_{i=1, \ldots, n}\left(\sigma \frac{d \rho_{i}}{d x}+\psi_{i}^{\prime} \rho_{i}\right)=0 \text { in } \Omega
$$

This suggests application of the Gronwall Lemma. It will be successful only in subintervals where all the $\psi_{i}^{\prime}$ are positive, and there are some by $(i i)$. So let us consider, for a fixed index $\nu$, the $\nu^{t h}$-equation of the system,

$$
\sigma \rho_{\nu}^{\prime \prime}+\psi_{\nu}^{\prime} \rho_{\nu}^{\prime}+\psi_{\nu}^{\prime \prime} \rho_{\nu}+a_{\nu \nu} \rho_{\nu}+\sum_{j \neq \nu} a_{\nu j} \rho_{j}=0 \text { in } I
$$


Equation (2.3) represents a balance between $\rho_{\nu}$ and the other $\rho_{j}$. As seen below in Step 3, since items in the $\sum$ are nonnegative, they can be discarded and (2.3) can be employed to find an upper bound for $\rho_{\nu}$ when $\psi_{\nu}$ is increasing. We can then exploit it to impede the growth of the other $\left\{\rho_{j}\right\}$. Namely, $\left\{\rho_{j}\right\}$ cannot be too large without forcing $\rho_{\nu}$ negative. But this can only be assured if the coupling is really there, namely if $a_{\nu j}>0$. This is the motivation for the ergodic type hypothesis in $(i v)$.

Given $\xi_{0}, 0<\xi_{0}<1-\lambda$, fix the period interval $\Lambda=\left[\xi_{0}, \xi_{0}+\lambda\right]$. We now limit our attention to $\Lambda$.

Select intervals $a+[-\delta, \delta]$ about zeros $a$ of the $\psi_{i}^{\prime}$. On the complement, there is a $k(\delta)>0$ with

$$
\psi_{i}^{\prime} \geqq k(\delta) \text { or } \psi_{i}^{\prime} \leqq-k(\delta) . i=1, \ldots, n
$$

Step 1: By $(i i)$, there is at least one interval $I_{0}=x_{0}+\left[-L_{0}, L_{0}\right] \subset \Lambda$ with

$$
\psi_{i}^{\prime} \geqq k(\delta) \text { in } I_{0}
$$

Then

$$
\frac{d}{d x} \sum_{i=1, \ldots, n} \rho_{i} \leqq-\frac{K_{0}}{2 \sigma} \sum_{i=1, \ldots, n} \rho_{i} \text { in } I_{0}
$$

and by Gronwall,

$$
\sum_{i=1, \ldots, n} \rho_{i}\left(x_{0}+L_{0}\right) \leqq e^{-\frac{K_{0}}{\sigma}} \sum_{i=1, \ldots, n} \rho_{i}\left(x_{0}-L_{0}\right)
$$

This is the exponential decay we are seeking. The remainder of the argument is to prove that (2.6) is not compromised when (2.5) does not hold.

Step 2: We check the zeros $a$ of the $\psi_{i}^{\prime}$. Although $\sum \rho_{i}$ may grow exponentially in these intervals, they are finite, say $N$, in number and we may choose them of small length. So restricting $\delta$,

$$
\sum_{i=1, \ldots, n} \rho_{i}(a+\delta) \leqq e^{\frac{M_{0}}{\sigma}} \sum_{i=1, \ldots, n} \rho_{i}(a-\delta)
$$

with, eg.,

$$
N M_{0} \delta<K_{0} L_{0}
$$

Also assume that $\delta \leqq \epsilon$ of $(i v)$. Now $\delta$ is fixed and in the sequel we suppress dependence of various constants on it.

Step 3: Both inequalities hold in (2.4) and we must exploit the coupling. Let $I=$ $[\alpha, \beta] \subset \Lambda$ be an interval where

$$
\begin{aligned}
& \psi_{i}^{\prime} \geqq k, i=1, \ldots, p, \\
& \psi_{j}^{\prime} \leqq-k, j=p+1, \ldots, n, \\
& |\beta-a|<\delta \leqq \epsilon \text { for some zero } a \text { of the } \psi_{k}^{\prime}, k=1, \ldots, n .
\end{aligned}
$$


Choose a favorable $\nu, \nu=1, \ldots, p$, from $(i i i)$, and assume that $(i v)$ holds, i.e., $a_{\nu j}>$ $0, j=p+1, \ldots, n$ in $\beta-\eta \leqq x \leqq \beta$, and consider the equation (2.3) for $\rho_{\nu}$. We can integrate this for a more convenient form, which gives us

$$
\begin{aligned}
\frac{d \rho_{\nu}}{d x}(x)= & \frac{d \rho_{\nu}}{d x}(\alpha) e^{-\frac{1}{\sigma}\left(\psi_{\nu}(x)-\psi_{\nu}(\alpha)\right)} \\
& -\frac{1}{\sigma} \int_{\alpha}^{x}\left\{\left(a_{\nu \nu}+\psi_{\nu}^{\prime}\right) \rho_{\nu}+\sum_{j \neq \nu} a_{\nu j} \rho_{j}\right\} e^{-\frac{1}{\sigma}\left(\psi_{\nu}(s)-\psi_{\nu}(\alpha)\right)} d s \text { in } I
\end{aligned}
$$

We shall use this in the two ways described at the beginning of the proof sketch. First, the sum in the right hand side of (2.9) is nonnegative, so we can omit it. Note also that $\psi_{\nu}$ is increasing so $\psi_{\nu}(x)-\psi_{\nu}(\alpha)>0$ in $I$. After some manipulation and applications of Gronwall, this leads to the upper bound

$$
\rho_{\nu}(x) \leqq K\left(\rho_{\nu}(\alpha)+\sigma\left|\rho_{\nu}^{\prime}(\alpha)\right|\right), x \in I
$$

Consequently, with

$$
C(\alpha)=\sum_{\nu=1, \ldots, p}\left(\rho_{\nu}(\alpha)+\sigma\left|\rho_{\nu}^{\prime}(\alpha)\right|\right),
$$

we obtain an upper bound for the favorable states which reads

$$
\sum_{\nu=1, \ldots, p} \rho_{\nu}(x) \leqq K C(\alpha), x \in I
$$

where $\mathrm{K}$ depends only on $\delta$ and the problem parameters.

Returning to (2.2), we then find the estimate

$$
\frac{d}{d x}\left(\rho_{p+1}+\cdots+\rho_{n}\right) \geqq-\frac{K_{1}}{\sigma} C(\alpha)+\frac{K_{2}}{\sigma}\left(\rho_{p+1}+\cdots+\rho_{n}\right) \text {, in } I
$$

This will tell us that if $\rho_{p+1}+\cdots+\rho_{n}$ is large at some $x^{*}$, it becomes exponentially larger for $x \geqq x^{*}$. We shall then show that this leads to a contradiction. In fact, we claim

$$
\rho_{p+1}+\cdots+\rho_{n} \leqq \frac{K_{1}}{K_{2}} C(\alpha) \text { in } I
$$

This will conclude the proof. Suppose that

$$
\left(\rho_{p+1}+\cdots+\rho_{n}\right)\left(x^{*}\right) \geqq \frac{K_{1}}{K_{2}} A C(\alpha) \text { with } A>1, \text { for some } x^{*} \in I \text {. }
$$

Integrating (2.12) then gives us that

$$
\begin{aligned}
\left(\rho_{p+1}+\cdots+\rho_{n}\right)(x) \geqq & \frac{K_{1}}{K_{2}} C(A-1) e^{\frac{K_{2}}{\sigma}\left(x-x^{*}\right)}+\frac{K_{1}}{K_{2}} C, x^{*}<x<\beta \\
& C=C(\alpha)
\end{aligned}
$$

Now, as foretold, we return to (2.9). Precisely, from $(i v), a_{\nu j}>0$ for $j=p+1, \ldots, n$, so there is a $\mu>0$ such that

$$
a_{\nu p+1} \rho_{p+1}+\cdots+a_{\nu n} \rho_{n} \geqq \mu\left(\rho_{p+1}+\cdots+\rho_{n}\right) \text { in }[\beta-\delta, \beta] .
$$


Using this and integrating (2.9), we find numbers $M, M_{1}$ and $M_{2}$ such that

$$
0 \leqq \frac{\rho_{\nu}(x)}{C(\alpha)} \leqq M_{1}-(A-1) M_{2} \delta \frac{K_{1}}{K_{2}} e^{\frac{M \delta}{\sigma}}, \text { in }[\beta-\delta, \beta]
$$

Now for $A>1,(2.16)$ cannot hold for small $\sigma$ since the right hand side becomes negative. Thus (2.13) holds for $\sigma$ sufficiently small.

The theorem now follows by concatening the three Steps.

3. Correlated and uncorrelated heads in a three state system. One of the simplest systems we may consider is a two state system for the unknown $\rho(x, t)=$ $\left(\rho_{1}(x, t), \rho_{2}(x, t)\right)$, a solution of (1.3) with $n=2$. Let us assume for this a configuration of potentials resembling (1.3) and a conformation change matrix

$$
A=\left(\begin{array}{rr}
-\alpha_{1} & \alpha_{2} \\
\alpha_{1} & -\alpha_{2}
\end{array}\right)
$$

where the support of the $\alpha_{i}$ is assumed to be a neighborhood of the minima of the potentials $\psi_{j}$. The conditions of Theorem 2.1 are satisfied, in particular, the ergodictype condition $(i v)$. The result of a sample simulation is given in Figure 3.1 and is a standard way to model conventional kinesin, cf [1], [4], [5]. The two heads are correlated.

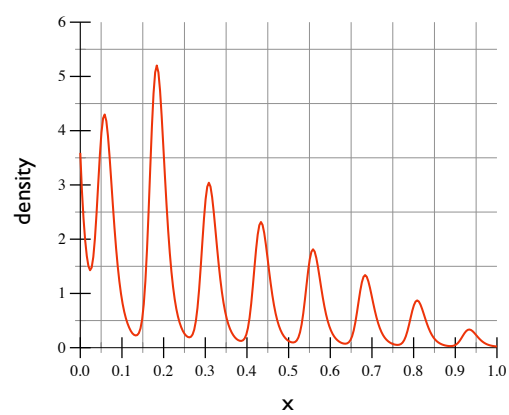

FIG. 3.1. Computed solution for two state rachet for interdigitated asymmetric potentials, period 4. Plot shows summed density $\rho_{1}+\rho_{2}$.

We may attempt a slightly increased degree of sophistication. The two heads of conventional kinesin are known to be connected by a rigid protein structure, called the neck linker, [29]. Hackney et al. in a recent experiment describe the decrease of transport properties of a version of kinesin (a chimera) fashioned by extending the neck linker through insertion of a spacer, rendering it more flexible, [9]. The two kinesin heads then fail to be correlated. Abstracting this situation, we may consider a three state system for conventional kinesin consisting of head-one, head-two, and the neck linker states. The neck linker does not participate in transport, so, in our 
framework, it is accounted for only in the conformation change matrix $A$. This will be a novel feature of the system, since we shall have only an ordinary differential equation for $\rho_{3}$. The two heads are not connected directly to each other but only to the neck linker. The system has the form

$$
\begin{aligned}
& \frac{\partial \rho_{i}}{\partial t}=\frac{\partial}{\partial x}\left(\sigma \frac{\partial \rho_{i}}{\partial x}+\psi_{i}^{\prime} \rho_{i}\right)+\sum_{j=1, \ldots, 3} a_{i j} \rho_{j} \text { in } \Omega, t>0, i=1,2 \\
& \frac{d \rho_{3}}{d t}=\sum_{j=1, \ldots, 3} a_{3 j} \rho_{j} \text { in } \Omega, t>0 \\
& \sigma \frac{\partial \rho_{i}}{\partial x}+\psi_{i}^{\prime} \rho_{i}=0 \text { on } \partial \Omega, t>0, i=1,2, \\
& \rho_{i} \geqq 0 \text { in } \Omega, \int_{\Omega}\left(\rho_{1}+\rho_{2}+\rho_{3}\right) d x=1, t>0 .
\end{aligned}
$$

where the $3 \times 3$ matrix $A$ has the generic representation

$$
A=\left(\begin{array}{lll}
* & 0 & * \\
0 & * & * \\
* & * & *
\end{array}\right)
$$

The stationary system for (3.2) is just

$$
\begin{aligned}
\frac{d}{d x}\left(\sigma \frac{d \rho_{i}}{d x}+\psi_{i}^{\prime} \rho_{i}\right)+ & \sum_{j=1, \ldots, 3} a_{i j} \rho_{j}=0 \text { in } \Omega, i=1,2 \\
\sum_{j=1, \ldots, 3} a_{3 j} \rho_{j} & =0 \text { in } \Omega \\
\sigma \frac{d \rho_{i}}{d x}+\psi_{i}^{\prime} \rho_{i} & =0 \text { on } \partial \Omega, i=1,2,
\end{aligned}
$$

Let us begin by revisiting the result about correlated two state systems in the present context. Thus we assume that all of the $*$ items in (3.3) do not vanish identically. In particular we assume that, in accord with (1.2),

$$
A=\left(\begin{array}{rrr}
a_{11} & 0 & a_{13} \\
0 & a_{22} & a_{23} \\
a_{31} & a_{32} & a_{33}
\end{array}\right)
$$

and

$$
\text { supp } a_{i j}=\text { a neighborhood of the minima of } \psi_{1} \text { and } \psi_{2}
$$


There are only 4 independent quantities in (3.5) owing to (1.2) and we assume that the supports of these functions are all equal. We may suppose that $\rho_{3}=0$ outside supp $a_{33}$ so that the last equation in (3.4) always makes sense. This can be guaranteed in the evolution system (3.2) by choice of suitable initial data. Eliminating $\rho_{3}$, we now find a new system for $\rho_{1}$ and $\rho_{2}$ alone of the form

$$
\begin{aligned}
\frac{d}{d x}\left(\sigma \frac{d \rho_{1}}{d x}+\psi_{1}^{\prime} \rho_{1}\right)-\alpha_{1} \rho_{1}+\alpha_{2} \rho_{2} & =0 \text { in } \Omega, i=1,2 \\
\frac{d}{d x}\left(\sigma \frac{d \rho_{2}}{d x}+\psi_{2}^{\prime} \rho_{2}\right)+\alpha_{1} \rho_{1}-\alpha_{2} \rho_{2} & =0 \text { in } \Omega, i=1,2 \\
\sigma \frac{d \rho_{i}}{d x}+\psi_{i}^{\prime} \rho_{i} & =0 \text { on } \partial \Omega, i=1,2,
\end{aligned}
$$

where, with the excruciating details, forms of $\alpha_{1}$ are

$$
\alpha_{1}=-\frac{a_{23}}{a_{33}} a_{31}=\frac{a_{23}}{a_{13}+a_{23}} a_{31}=-a_{11} \frac{a_{23}}{a_{13}+a_{23}}=-a_{11}\left(1-\frac{a_{13}}{a_{13}+a_{23}}\right) \geqq 0
$$

and similarly for $\alpha_{2}$. The new system (3.7) for just $\rho_{1}$ and $\rho_{2}$ satisfies the conditions of Theorem 2.1. Thus the conclusion of the theorem applies, and we recapture our prior result. The result of a simulation is given in Figure 3.2 on the left.
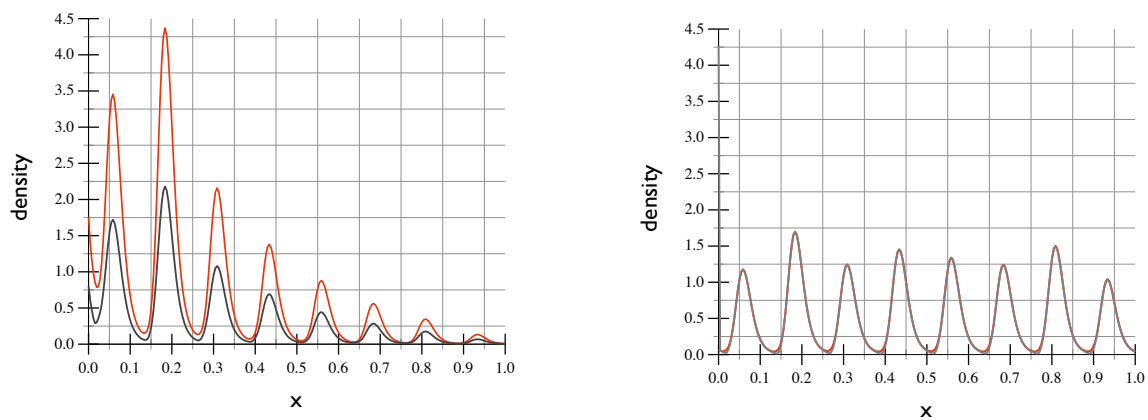

FIG. 3.2. Two state rachet with neck linker with heads correlated (left) and heads uncorrelated (right). Red plot is the sum of the head densities $\rho_{1}+\rho_{2}$ and gray plot is the neck linker density $\rho_{3}$. The left plot illustrates transport. In the uncorrelated example, there is no transport and it turns out that $\rho_{3}=\rho_{1}+\rho_{2}$.

We can decorrellate the heads. The idea, obviously, is to choose the $\left\{a_{i j}\right\}$ so that (3.4) decouples. So assume in (3.5) that

$$
\begin{aligned}
& \Lambda_{1}=\operatorname{supp} a_{11}=\operatorname{supp} a_{13}=\text { a neighborhood of the minima of } \psi_{1} \\
& \Lambda_{2}=\operatorname{supp} a_{22}=\operatorname{supp} a_{23}=\text { a neighborhood of the minima of } \psi_{2} \text {, and } \\
& \Lambda_{1} \cap \Lambda_{2}=\emptyset
\end{aligned}
$$

Note now that from (3.8), $\alpha_{1}=0$ and the same for $\alpha_{2}$. 
We find for the system (3.4), that

$$
\begin{aligned}
& \frac{d}{d x}\left(\sigma \frac{d \rho_{i}}{d x}+\psi_{i}^{\prime} \rho_{i}\right)=0 \text { in } \Omega, i=1,2 \\
& \rho_{3}=\rho_{1} \text { or } \rho_{2} \text { or } 0 \text { in } \Omega \\
& \sigma \frac{d \rho_{i}}{d x}+\psi_{i}^{\prime} \rho_{i}=0 \text { on } \partial \Omega, i=1,2
\end{aligned}
$$

which is the same except for mass fractions, cf. (1.10), as writing that

$$
\rho_{1}=\rho_{1}^{\sharp} \text { and } \rho_{2}=\rho_{2}^{\sharp}
$$

where there is no transport. The result of a simulation is given in Figure 3.2 on the right.

\section{REFERENCES}

[1] Ajdari, A. And Prost, J. (1992) Mouvement induit par un potentiel périodique de basse symétrie: dielectrophorese pulse, C. R. Acad. Sci. Paris t. 315, Série II, 1653.

[2] Astumian, R.D. (1997) Thermodynamics and kinetics of a Brownian motor, Science 276 (1997), 917-922.

[3] Benamou, J.-D. and Brenier, Y. (2000) A computational fluid mechanics solution to the Monge-Kantorovich mass transfer problem, Numer. Math. 84, 375-393.

[4] Chipot, M., Hastings, S., and Kinderlehrer, D., Transport in a molecular motor system (2004) Math. Model. Numer. Anal. (M2AN) 38 no. 6, 1011-1034

[5] Chipot, M., Kinderlehrer, D. and KowalczyK, M. (2003) A variational principle for molecular motors, Meccanica, 38, 505-518

[6] Doering, C., Ermentrout, B. And Oster, G. (1995) Rotary DNA motors. Biophys. J. 69(6), $2256-67$

[7] Dolbeault, J., Kinderlehrer, D., and Kowalczyk, M. Remarks about the flashing rachet, to appear Proc. PASI 2003

[8] Hackney, D.D. (1996) The kinetic cycles of myosin, kinesin, and dynein, Ann. Rev. Physiol., $\mathbf{5 8}, 731-750$

[9] Hackney, D.D., Stock, M. F., Moore, J., and Patterson, R. (2003) Modulation of kinesin half-site ADP release and kinetic processvity by a spacer between the head grounps, Biochem., 42, 12011 - 12018

[10] Hastings, S. AND Kinderlehrer, D. (2005) Remarks about diffusion mediated transport: thinking about motion in small systems, Nonconvex Optim. Appl., Springer, 79, 497-511

[11] Hastings, S., Kinderlehrer, D. And McLeod, J.B. Diffusion mediated transport in multiple state systems, to appear

[12] Howard, J. (2001) Mechanics of Motor Proteins and the Cytoskeleton, Sinauer Associates, Inc.

[13] Huxley, A.F. (1957) Muscle structure and theories of contraction, Prog. Biophys. Biophys. Chem. 7, 255-318.

[14] Jordan, R., Kinderlehrer, D. And Otto, F. (1998) The variational formulation of the Fokker-Planck equation, SIAM J. Math. Anal. Vol. 29 no. 1, 1-17.

[15] Kinderlehrer, D. And KowalczyK, M (2002) Diffusion-mediated transport and the flashing ratchet, Arch. Rat. Mech. Anal. 161, 149-179.

[16] Kinderlehrer, D. And Walkington, N. (1999) Approximation of parabolic equations based upon Wasserstein's variational principle, Math. Model. Numer. Anal. (M2AN) 33 no. 4, $837-852$.

[17] Magnasco, M. (1993) Forced thermal rachets, Phys. Rev. Lett. 67, 1477-1481

[18] Okada, Y. ANd Hirokawa, N. (1999) A processive single-headed motor: kinesin superfamily protein KIF1A, Science Vol. 283, 19

[19] OKada, Y. AND HiRokawa, N. (2000) Mechanism of the single headed processivity: diffusional anchoring between the K-loop of kinesin and the $\mathrm{C}$ terminus of tubulin, Proc. Nat. Acad. Sciences 7 no. 2, 640-645.

[20] Отто, F. (1998) Dynamics of labyrinthine pattern formation: a mean field theory, Arch. Rat. Mech. Anal. 141, 63-103 
[21] Отто, F. (2001) The geometry of dissipative evolution equations: the porous medium equation, Comm. PDE 26, 101-174

[22] Parmeggiani,A., Jülicher, F., Ajdari, A. And Prost, J. (1999) Energy transduction of isothermal ratchets: generic aspects and specific examples close and far from equilibrium, Phys. Rev. E, 60 no. 2, 2127-2140.

[23] Perthame, B. The general relative entropy principle

[24] Peskin, C.S.. Ermentrout, G.B. And Oster, G.F. (1995) The correlation ratchet: a novel mechanism for generating directed motion by ATP hydrolysis, in Cell Mechanics and Cellular Engineering (V.C Mow et.al eds.), Springer, New York

[25] Protter, M. And Weinberger, H.(1967) Maximum principles in differential equations, Prentice Hall, Englewood Cliffs, N. J.

[26] Purcell, E. M.,(1971) Life at low Reynolds' Number Am. J. Phys. 45, 3-11

[27] Reimann, P. (2002) Brownian motors: noisy transport far from equilibrium, Phys. Rep. 361 nos. 2-4, 57-265.

[28] Schliwa, M., ED (2003) Molecular Motors, Wiley-VCH Verlag, Wennheim

[29] Vale, R.D. And Milligan, R.A. (2000) The way things move: looking under the hood of motor proteins, Science 288, 88-95.

[30] Villani, C (2003) Topics in optimal transportation, AMS Graduate Studies in Mathematics vol. 58, Providence

[31] Zeidler, E (1986) Nonlinear functional analysis and its applications, I Springer, New York, N.Y. 